\title{
Integrated Energy Method for Propulsion Dynamics Analysis of Air-Pressurized Waterjet Rocket
}

\author{
By Hsing-Juin LEE, Chih-Hong CHIU and Wen-Kung HsiA \\ Department of Mechanical Engineering, National Chung-Hsing University, Taichung 40227, Taiwan, Republic of China
}

(Received April 7th, 2000)

\begin{abstract}
The launching of a waterjet rocket has been a very popular idea in recent years. Its basic propulsion principle makes use of the high-pressurized air inside the rocket's main body to swiftly expel the water out of the nozzle and thus generate thrust. The waterjet rocket is characterized with nature, interest, combustionlessness, environmental friendliness, simplicity, and minimal cost. Moreover, it is a very good science model for propulsion analysis, design, experiment, and education because of an abundance of easily adjustable key parameters. This model also features separately stored energy and mass of the propellant, in contrast to a conventional rocket. However, related literature shows that no indepth theoretical analysis of the waterjet rocket has been attempted for various reasons. In this research, the propulsion dynamics of a waterjet rocket is analyzed by simultaneously solving the momentum and the newly derived generalized power equations to predict its flight histogram, computationally, and convolutionally. This integrated energy approach synthesizes the internal and external dynamics analyses together and ingeniously takes full advantage of the clear power supply of pressurized air in a waterjet rocket. The analysis results are generally agreeable with the experimental flight data. While the new power equation herein gives a complete spectrum of physical parameters to be manipulated, there will be wider room in quest of better rocket propulsion performance, especially through the heuristic research of this versatile but affordable waterjet rocket.
\end{abstract}

Key Words: Waterjet Rocket, Interweaved Transport Analysis, Generalized Equations, Integrated Energy Method, Flight Dynamics, Versatile Propulsion Model

\section{Nomenclature}

$\sum F_{\text {ext.surf }}$ : external force component acting on the external hard surface of the rocket along its centerline (excluding $A_{\mathrm{e}}$ )

$\rho:$ mass density

$\rho_{\mathrm{a}}:$ air mass density

$\rho_{\mathrm{w}}:$ water mass density

$A$ : frontal area of waterjet rocket

$\bar{A}:$ area vector

$A_{\mathrm{e}}$ : nozzle exit area for rocket

$C S_{\text {comoving }}$ : comoving control surface

$C V_{\text {comoving }}$ : comoving control volume

$C_{\mathrm{D}}$ : aerodynamic drag coefficient

$C_{\mathrm{p}}:$ constant-pressure specific heat

$C_{\mathrm{v}}:$ constant-volume specific heat

$D\left(E_{\mathrm{ksyst}}\right) / D t$ : material derivative of kinetic energy of the rocket mass system with respect to time

$F:$ force

$M(t)$ : time-varying mass of the rocket

$P_{\mathrm{a}}$ : atmospheric pressure

$P_{\mathrm{e}}$ : pressure intensity on the rocket nozzle exit area

$P_{\mathrm{i}}: \quad$ air pressure in the waterjet rocket

$V_{\mathrm{cv}}$ : absolute rocket velocity

$\dot{W}$ : time rate of output work by the rocket mass system

$e_{\mathrm{k}}:$ kinetic energy per unit of mass

$g(t)$ : gravitational acceleration at an instant

$k$ : ratio between $C_{\mathrm{p}}$ and $C_{\mathrm{v}}$

$m_{\mathrm{i}}$ : instantaneous mass of water in the waterjet rocket

$\dot{m}_{\mathrm{e}}$ : outlet mass flow rate

$t:$ time

$v_{\mathrm{e}}$ : outlet propellant velocity relative to the rocket

$v_{\text {rel }}:$ velocity relative to the rocket

$\forall:$ volume

$\forall_{\mathrm{a}}: \quad$ volume of air in the waterjet rocket

\section{Introduction}

The launching of a waterjet rocket has been a very popular idea in recent years (Figs. 1, 2), especially in Japan and some other countries. This type of rocket generally may use a rocket-shaped vessel, such as a PET bottle commonly used for carbonated drinks with a volume of 1 or 2 liters. The bottle should be filled partly with water and partly with highpressurized air (Fig. 3) before launch. The high-pressurized air will swiftly push the inside water out of the nozzle and thus generate thrust for the rocket's main body. Because of the relatively higher density of ejected water compared with the gaseous exhaust of a traditional rocket, ${ }^{1-3)}$ the thrust gen- 


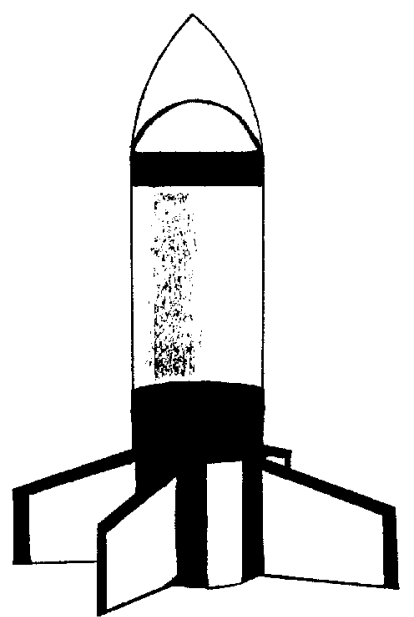

Fig. 1. Sample of waterjet rocket.

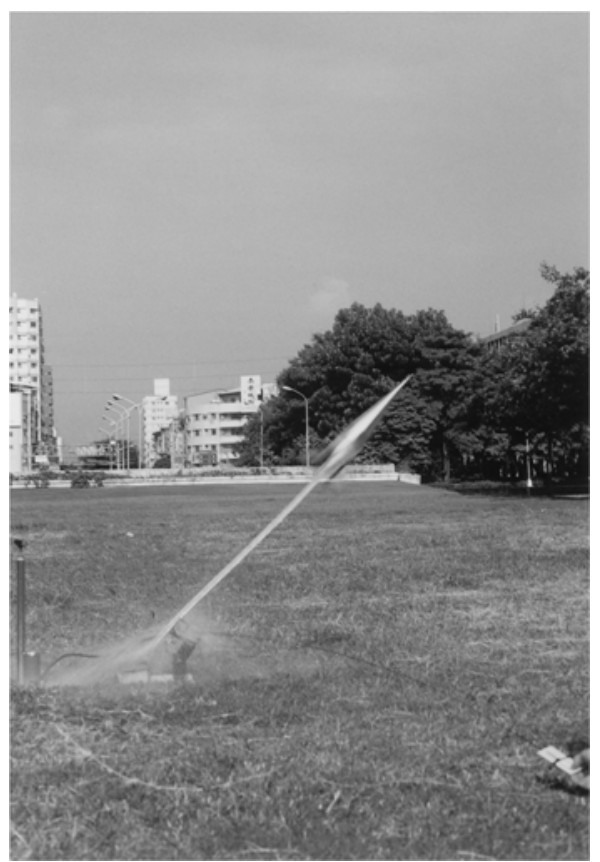

Fig. 2. Launching of waterjet rocket.

erated by a waterjet rocket can easily propel the rocket to a speed exceeding $40 \mathrm{~m} / \mathrm{sec}$, even when the relative velocity of ejected water is comparatively low. This type of rocket has the following characteristics:

$* * *$ Much simpler structure compared with other types of rockets.

$* * *$ No combustion (thus no combustion chamber). This eliminates the need for heat-resistant material for the rocket's main body and nozzle. Moreover, no smoke or heat can be detected (comparing with the related risk of shoulder-launched antitank missiles for example).

$* * *$ Separately stored and independently adjustable energy and mass of the propellant in contrast to conventional propellant. These adjustabilities are valuable for affordable propulsion science research.
External Surface Force

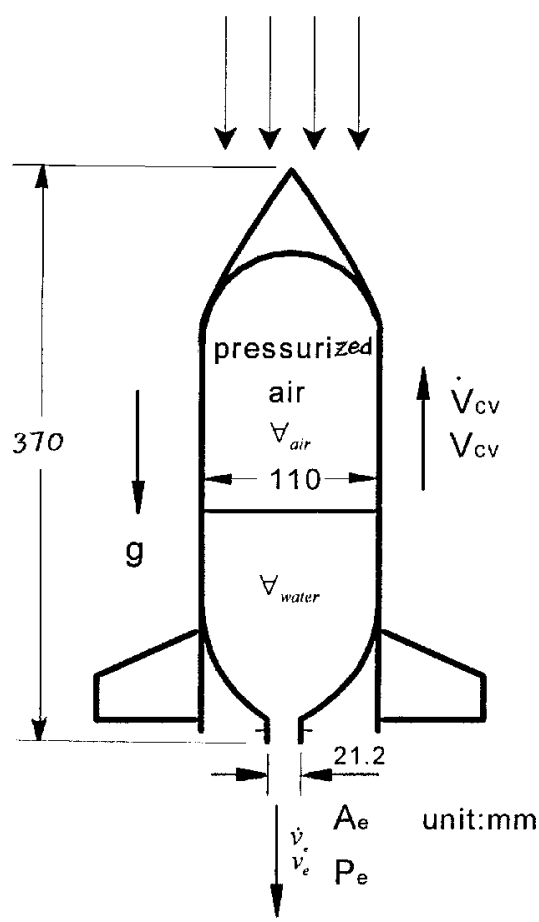

Fig. 3. Schematics for waterjet rocket.

$* * *$ Interesting !

$* * *$ Minimal cost involved in manufacture, launch, and associated equipment, thus making acquisition and establishment easy.

$* * *$ Very good model for propulsion analysis, design, experiment, and education because of an abundance of very easily adjustable key parameters, such as internal air pressure, air/water proportion, shell mass, shell diameter (uses different bottle), nozzle size/shape, configurations, and associated $C_{\mathrm{d}}$, launch angle, and so on.

Nevertheless, related literature ${ }^{2-7)}$ shows that no in-depth theoretical analysis of the waterjet rocket has been attempted. Perhaps, one reason for the untamed research area is due to the generalized total kinetic power equation for rocket having been derived only recently. ${ }^{8)}$ In this research, the propulsion dynamics of a waterjet rocket is analyzed by solving the momentum and energy equations simultaneously to predict its flight histogram. Herein a more direct and natural lagrangian Reynolds transport equation (LRTE) ${ }^{8)}$ instead of the traditional one ${ }^{9)}$ will be employed. The LRTE is specifically suitable for the analysis of jet propulsion dynamics, such as deriving the rocket equation of motion in this section and the sophisticated rocket total kinetic power later. To highlight the analysis process and to avoid kinematic complication therefore in the following discussion, the waterjet rocket is supposed to be launched vertically. It is now timely to briefly review the derivation of the unabridged rocket momentum equation, nevertheless here by the highly efficient interweaved procedure with control volume enclos- 


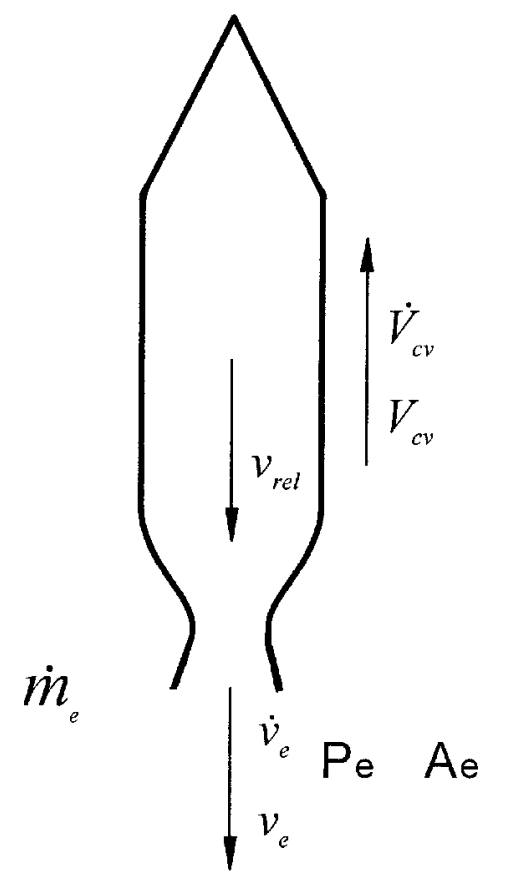

Fig. 4. Schematics for a general rocket engine.

ing and comoving with a general rocket in flight (Fig. 4). In that light, we first separate the basic momentum equation into two parts involving $V_{\mathrm{cv}}$ and $v_{\text {rel }}$ respectively, and then invoke the lagrangian Reynolds transport analysis for $v_{\text {rel }}$ part only amid the deriving process

$$
\begin{aligned}
\sum & F_{\text {ext.surf }}+\left(P_{\mathrm{e}} A_{\mathrm{e}}\right)-M(t) g(t) \\
= & \frac{D(\text { momentum })_{\text {syst }}}{D t} \\
= & \frac{D}{D t} \iiint_{S M}\left(V_{\mathrm{cv}}-v_{\mathrm{rel}}\right) \rho d \forall \\
= & \frac{D}{D t}\left[V_{\mathrm{cv}} \iiint_{S M} \rho d \forall\right]-\frac{D}{D t} \iiint_{S M} v_{\text {rel }} \rho d \forall \\
= & {\left[\frac{D}{D t} V_{\mathrm{cv}}\right] M+V_{\mathrm{cv}}\left[\frac{D}{D t} M\right]-\frac{\partial}{\partial t} \iiint_{C V_{\text {comoving }}} v_{\text {rel }} \rho d \forall } \\
& -\iint_{C S_{\text {comoving }}} v_{\text {rel }} \rho \bar{v}_{\text {rel }} \cdot d \bar{A} \\
= & M \dot{V}_{\mathrm{cv}}-\frac{\partial}{\partial t} \iiint_{C V_{\text {comoving }}} v_{\text {rel }} \rho d \forall-\left(\dot{m}_{\mathrm{e}} v_{\mathrm{e}}\right)
\end{aligned}
$$

where $S M$ represents the system mass of rocket, whereas, $\sum F_{\text {ext.surf }}$ is assumed forward positive as usual. The high efficiency of deriving the above Eq. (1) by the interweaved procedure with LRTE is very clear. In a low altitude flight with appreciable atmospheric pressure, as for a waterjet rocket, the gauge pressure and force can be used accordingly. Also, $C S$ is the comoving control surface enclosing the comoving control volume $C V$, which is the rocket in flight. It is noteworthy that the term involving material derivative of system mass $M$ naturally disappears in the above processing because of the conservation of mass.

Equation (1) can be rearranged as

$$
\begin{gathered}
\left(\sum F_{\text {ext.surf }}\right)+P_{\mathrm{e}} A_{\mathrm{e}}-M(t) g(t)+\dot{m}_{\mathrm{e}} v_{\mathrm{e}} \\
=\dot{V}_{\mathrm{cv}} M(t)-\frac{\partial}{\partial t} \iiint_{C V_{\text {comoving }}} v_{\text {rel }} \rho d \forall .
\end{gathered}
$$

This is one of the two equations prepared for propulsion analysis of a waterjet rocket in section 3 .

\section{Interweaved Transport Analysis of the Generalized Rocket Total Kinetic Power}

Instead of the relatively entangling derivation by the direct transport analysis approach in Ref. 8, however we herein derive the generalized equation for rocket total kinetic power by the highly efficient interweaved procedure, again with control volume enclosing the rocket in flight. In that light, we first separate the kinetic power equation into several parts, then use the transport analysis only for the two parts involving $v_{\text {rel }}$ and $v_{\text {rel }}^{2}$ amid the deriving process in the following manner

$$
\begin{aligned}
& \frac{D\left(E_{\mathrm{ksyst}}\right)}{D t}+\dot{W} \\
& =\frac{D}{D t} \iiint_{S M} \frac{1}{2}\left(V_{\mathrm{cv}}-v_{\mathrm{rel}}\right)^{2} \rho d \forall+\dot{W} \\
& =\frac{D}{D t} \iiint_{S M} \frac{1}{2}\left(V_{\mathrm{cv}}\right)^{2} \rho d \forall-\frac{D}{D t} \iiint_{S M} V_{\mathrm{cv}} v_{\mathrm{rel}} \rho d \forall \\
& \quad+\frac{D}{D t} \iiint_{S M} \frac{1}{2}\left(v_{\mathrm{rel}}\right)^{2} \rho d \forall+\dot{W}
\end{aligned}
$$

This equation can be further processed as

$$
\begin{aligned}
& \frac{D\left(E_{\mathrm{ksyst}}\right)}{D t}+\dot{W} \\
& =V_{\mathrm{cv}}\left[\dot{V}_{\mathrm{cv}} M(t)\right]-\dot{V}_{\mathrm{cv}} \iiint_{S M} v_{\mathrm{rel}} \rho d \forall \\
& -V_{\mathrm{cv}} \frac{D}{D t} \iiint_{S M} v_{\mathrm{rel}} \rho d \forall+\frac{D}{D t} \iiint_{S M} \frac{1}{2}\left(v_{\mathrm{rel}}\right)^{2} \rho d \forall+\dot{W}
\end{aligned}
$$

Then, by LRTE the above equation becomes

$$
\frac{D\left(E_{\mathrm{ksyst}}\right)}{D t}+\dot{W}
$$




$$
\begin{aligned}
& =V_{\mathrm{cv}}\left[\dot{V}_{\mathrm{cv}} M(t)-\frac{\partial}{\partial t} \iiint_{C V_{\text {comoving }}} v_{\text {rel }} \rho d \forall\right] \\
& -\dot{V}_{\mathrm{cv}} \iiint_{C V_{\text {comoving }}} v_{\text {rel }} \rho d \forall+\frac{\partial}{\partial t} \iiint_{C V_{\text {comoving }}} \frac{1}{2}\left(v_{\text {rel }}\right)^{2} \rho d \forall \\
& -V_{\mathrm{cv}}\left(v_{\mathrm{e}} \dot{m}_{\mathrm{e}}\right)+\frac{1}{2}\left(\dot{m}_{\mathrm{e}} v_{\mathrm{e}}^{2}\right)+\dot{W}
\end{aligned}
$$

Furthermore, the kinetic power output to the environment by the rocket mass system can be expressed as

$$
\begin{aligned}
& \dot{W}=\left(-\sum F_{\text {ext.surf }}\right) V_{\mathrm{cv}}-P_{\mathrm{e}} A_{\mathrm{e}}\left(V_{\mathrm{cv}}-v_{\mathrm{e}}\right) \\
& +\iiint_{C V_{\text {comoving }}} g(t)\left(V_{\mathrm{cv}}-v_{\mathrm{rel}}\right) \rho d \forall \\
& =\left(-\sum F_{\text {ext.surf }}\right) V_{\mathrm{cv}}-P_{\mathrm{e}} A_{\mathrm{e}}\left(V_{\mathrm{cv}}-v_{\mathrm{e}}\right) \\
& +\left[V_{\mathrm{cv}}(g(t) M(t))-g(t) \iiint_{C V_{\text {comoving }}} v_{\text {rel }} \rho d \forall\right]
\end{aligned}
$$

Substituting Eq. (2) for the right hand side bracketed terms of Eq. (5), and substituting Eq. (6) for $\dot{W}$ in Eq. (5), after some algebraic manipulations and canceling, the total kinetic power produced by the rocket engine can be obtained as

$$
\begin{aligned}
& \frac{D\left(E_{\text {ksyst }}\right)}{D t}+\dot{W} \\
& =P_{\mathrm{e}} A_{\mathrm{e}} v_{\mathrm{e}}-g(t) \iiint_{C V_{\text {comoving }}} v_{\text {rel }} \rho d \forall \\
& \quad-\dot{V}_{\mathrm{cv}} \iiint v_{C V_{\text {comoving }}} \rho d \forall \\
& \quad+\frac{\partial}{\partial t} \iiint \int_{C V_{\text {comoving }}} \frac{1}{2}\left(v_{\text {rel }}\right)^{2} \rho d \forall+\frac{1}{2} \dot{m}_{\mathrm{e}} v_{\mathrm{e}}^{2} .
\end{aligned}
$$

It is important to note that after complicated mathematical manipulation, Eq. (7) clearly shows that all the velocities involved are relative quantities, thus avoiding the possibility of violating the energy conservation law. Alternately, we can say that the rocket total kinetic power should be invariant to any unaccelerating observer. It is noteworthy that both Eqs. (2) and (7) involve unknown variables $\dot{V}_{\mathrm{cv}}$ and $\dot{v}_{\mathrm{e}}$, explicitly or implicitly.

\section{Method for Integrated Propulsion Dynamics Analy- ses of Waterjet Rocket}

In this study, the propulsion dynamics of a waterjet rocket is analyzed by simultaneously solving the momentum and the new generalized total kinetic power equations ${ }^{8)}$ by computational procedure with the aid of Matlab. Then we can manage to obtain the instant $\dot{V}_{\mathrm{cv}}$ and $\dot{v}_{\mathrm{e}}$, and convolutionally in this manner we can solve for the whole flight histogram of the waterjet rocket. This integrated energy method synthesizes both internal and external dynamics analyses of the waterjet rocket. Moreover, this approach ingeniously takes full advantage of the predictable power supply in an isentropic process of the pressurized air in a waterjet rocket. Also, for better apex prediction of the waterjet rocket, the effect of pure air expulsion post waterjet is generally considered for the waterjet rocket.

In the momentum equation, the aerodynamic drag force on the hard surface $\sum F_{\text {ext.surf }}$ at a given moment can be expressed by

$$
\sum F_{\text {ext.surf }}=\frac{1}{2} \rho_{a} V_{\mathrm{cv}}{ }^{2} A C_{\mathrm{D}}
$$

For a given waterjet rocket, the aerodynamic drag force on the hard surface versus its flight velocity $V_{\mathrm{cv}}$ can be readily obtained by an experimental method. Then the associated $C_{\mathrm{D}}$ can be calculated accordingly. In this study, the drag coefficient of the specific waterjet rocket as shown in Fig. 3 and specified in section 4 is experimentally obtained as 0.52 .

Meanwhile, the total kinetic power of the waterjet rocket can be easily calculated by recognizing the $\dot{m}_{\mathrm{e}} / \rho_{\mathrm{w}}$ as the volume expansion rate of internal compressed air (where $\rho_{\mathrm{w}}$ is the mass density of the water), since $\dot{m}_{\mathrm{e}} / \rho_{\mathrm{w}}=A_{\mathrm{e}} v_{\mathrm{e}}$. Thus with known $v_{\mathrm{e}}$ at a given instant we can easily calculate the total kinetic power of the left side of Eq. (7) at that instant

$$
\begin{aligned}
& \frac{D\left(E_{\mathrm{ksyst}}\right)}{D t}+\dot{W} \\
& \quad=p_{\mathrm{i}}\left(\dot{m}_{\mathrm{e}} / \rho_{\mathrm{w}}\right)=p_{\mathrm{i}} A_{\mathrm{e}} v_{\mathrm{e}}
\end{aligned}
$$

It is obvious that the internal air pressure $p_{\mathrm{i}}$ will drop while the internal air volume expands according to thermodynamic law. For a relatively short duration of waterjet flight (say a powered phase of a fractional second with negligible heat transfer), it is reasonable to assume this expansion as an isentropical process. Therefore the internal air pressure between two time stations elapsed by a short duration can be expressed as

$$
p_{\mathrm{i}}(t) \forall_{\mathrm{a}}{ }^{k}(t)=p_{\mathrm{i}}(t+\delta t) \forall_{\mathrm{a}}{ }^{k}(t+\delta t)
$$

where $k$ is the specific heat ratio between constant-pressure specific heat $C_{\mathrm{p}}$ and constant-volume specific heat $C_{\mathrm{v}}$. And

$$
\forall_{\mathrm{a}}(t+\delta t)=\forall_{\mathrm{a}}(t)+A_{\mathrm{e}} v_{\mathrm{e}} \delta t
$$

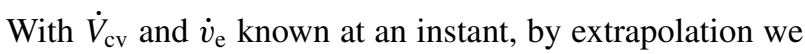
can then calculate the $V_{\mathrm{cv}}$ and $v_{\mathrm{e}}$ at the next time station. Moreover, with rocket velocity $V_{\mathrm{cv}}$ being known at the next instant, we can easily calculate the associated aerodynamic drag force on the hard surface $\sum F_{\text {ext.surf }}$ for a given waterjet rocket at that instant. Thus the momentum equation 2 is ready again for simultaneous solution with total kinetic power equation 7 to obtain the new $\dot{V}_{\mathrm{cv}}$ and $\dot{v}_{\mathrm{e}}$ for convolutional calculation. By this methodology, with known $V_{\mathrm{cv}}$ for every instant we can easily calculate the whole flight histogram of the waterjet rocket (and corresponding gravity, if necessary). 


\section{Numerical Analysis and Result}

Except for the initial time station zero, we can use the calculation strategy stated above. For time station zero, since the generalized power equation is nil, we must turn to a basic force analysis method for estimating the initial $\dot{V}_{\mathrm{cv}}$ for the waterjet rocket. This estimation is done by considering the internal air pressure and the internal fluid inertia. After substituting this estimated initial $\dot{V}_{\text {cv }}$ in the momentum equation, we can estimate the $\dot{v}_{\mathrm{e}}$ and subsequently other relevant data. As long as the time step is small enough for the specific waterjet rocket used as described in this section later, a $0.0001 \mathrm{sec}$ time step will be small enough to avoid any significant initial condition interference with the flight histogram and apex of the launching waterjet rocket. Fortunately from time station one on, the strategy will be back to the normal procedure of solving simultaneous equations of momentum and total kinetic power as explained in section 3 .

Where the following term in the momentum Eq. (2)

$$
-\frac{\partial}{\partial t} \iiint_{C V_{\text {comoving }}} v_{\text {rel }} \rho d \forall
$$

shall be further processed in order to be in terms of $v_{\mathrm{e}}$ and unknown variables $\dot{v}_{\mathrm{e}}$

$$
\begin{aligned}
& -\iiint_{C V_{\text {comoving }}}\left(\frac{\partial}{\partial t} v_{\text {rel }}\right) \rho d \forall-\iiint_{C V_{\text {comoving }}} v_{\text {rel }} \frac{\partial}{\partial t}(\rho d \forall) \\
& =-\alpha(t) \dot{v}_{\mathrm{e}} m_{\mathrm{i}}+\beta(t) v_{\mathrm{e}} \dot{m}_{\mathrm{e}}
\end{aligned}
$$

where the time-varying $\alpha(t)$ and $\beta(t)$ are ratios that can be obtained by comparing corresponding terms of the above equation, whereas the $m_{\mathrm{i}}$ is the mass of water in the waterjet rocket at an instant. Also, since for uniform tubular nozzle of the waterjet rocket used in this study there is no convective acceleration at the outlet, only the local acceleration remains; thus $\dot{v}_{\mathrm{e}}$ equates to $\frac{\partial}{\partial t} v_{\mathrm{e}}$ under this condition.

The following term in the total kinetic power Eq. 7

$$
\frac{\partial}{\partial t} \iiint_{C V_{\text {comoving }}} \frac{1}{2}\left(v_{\text {rel }}\right)^{2} \rho d \forall
$$

shall be further processed to be in terms of unknown variable $\dot{v}_{\mathrm{e}}$

$$
\begin{gathered}
\iiint_{C V_{\text {comoving }}} v_{\text {rel }}\left(\frac{\partial}{\partial t} v_{\text {rel }}\right) \rho d \forall+\iiint_{C V_{\text {comoving }}} \frac{1}{2}\left(v_{\text {rel }}\right)^{2} \frac{\partial}{\partial t}(\rho d \forall) \\
\quad=\gamma(t) v_{\mathrm{e}} \dot{v}_{\mathrm{e}} m_{\mathrm{i}}-\mu(t)\left[\frac{1}{2}\left(v_{\mathrm{e}}\right)^{2} \dot{m}_{\mathrm{e}}\right]
\end{gathered}
$$

where the time-varying $\gamma(t)$ and $\mu(t)$ are ratios that can be obtained by comparing corresponding terms of the above equation.

The schematics of a waterjet rocket has been shown in Fig. 3, for example. The following data are referred for the Matlab program (see Appendix) to calculate its flight histogram: air density: $\rho_{\mathrm{a}}=1.23 \mathrm{~kg} / \mathrm{m}^{3}$

water density: $\rho_{\mathrm{w}}=1,000 \mathrm{~kg} / \mathrm{m}^{3}$

atmospheric pressure: $P_{\mathrm{a}}=101,300 \mathrm{~N} / \mathrm{m}^{2}$

aerodynamic drag coefficient: $C_{\mathrm{D}}=0.52$,

inner cross-section area of waterjet rocket: $A_{\mathrm{i}}=$ $0.0095 \mathrm{~m}^{2}$

inner cross-section area of nozzle: $A_{\mathrm{e}}=0.00035 \mathrm{~m}^{2}$

shell thickness (in general $)=0.0004 \mathrm{~m}(\mathrm{PET})$

shell mass (waterjet rocket without water and air) $=$ $0.255 \mathrm{~kg}$

volume of water in the waterjet rocket $=0.0009 \mathrm{~m}^{3}$

volume of pressurized air in the waterjet rocket $=$ $0.0011 \mathrm{~m}^{3}$

initial total mass of the waterjet rocket $=1.164 \mathrm{~kg}$

$P_{\mathrm{e}}=101,300 \mathrm{~N} / \mathrm{m}^{2}$

air pressure in the waterjet rocket: $P_{\mathrm{i}}=7 \times 101,300 \mathrm{~N} / \mathrm{m}^{2}$ time step: $\Delta t=0.0001 \mathrm{sec}$

The numerical results are very interesting. The lowest curve in Fig. 5 shows the flight apex versus the shell mass of the given waterjet rocket for given internal air pressure and air/water ratio. This figure indicates that an optimal height will happen for shell mass around 0.20 to $0.30 \mathrm{~kg}$ under the above given internal air pressure and other conditions. For example, the calculated flight height for $0.255 \mathrm{~kg}$ shell mass is $54.5 \mathrm{~m}$, which is generally agreeable with the corresponding experimental result of $51 \mathrm{~m}$. Understandably, an even lighter shell mass will not give a higher value for the same reason that even a muscular giant cannot throw a light straw far. This phenomenon is due to the complicated interaction of aerodynamic drag force and kinetic energy owned by the shell mass.

Figure 5 also shows the flight apexes versus the shell mass for other internal air pressures in the given waterjet rocket for a definite air/water volume ratio. This clearly shows that a higher internal pressure will give a higher apex for a given shell mass and air/water ratio. This situation is quite reasonable, since higher internal air pressure will usually give higher kinetic energy for the rocket's main body. All computations in this study are processed on a common laboratory

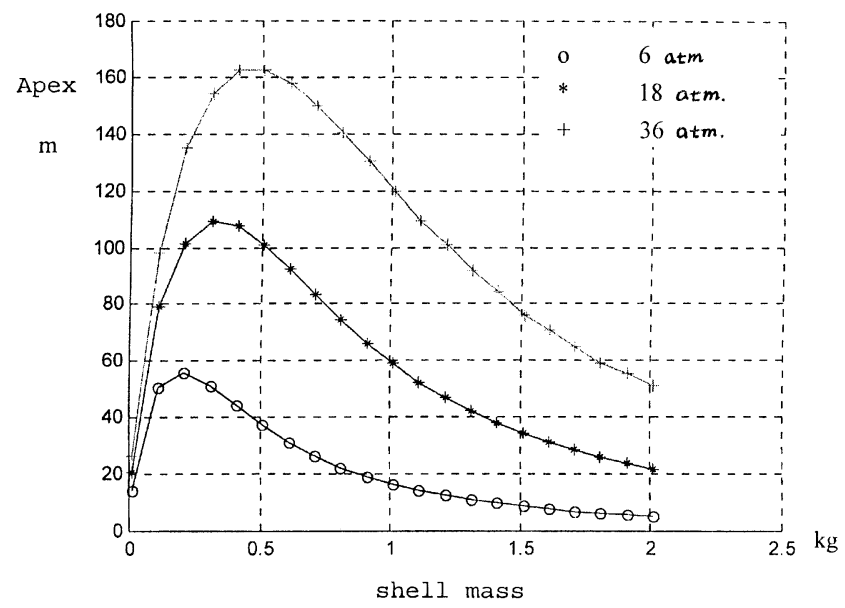

Fig. 5. Apex vs. shell mass for various internal air pressures. 


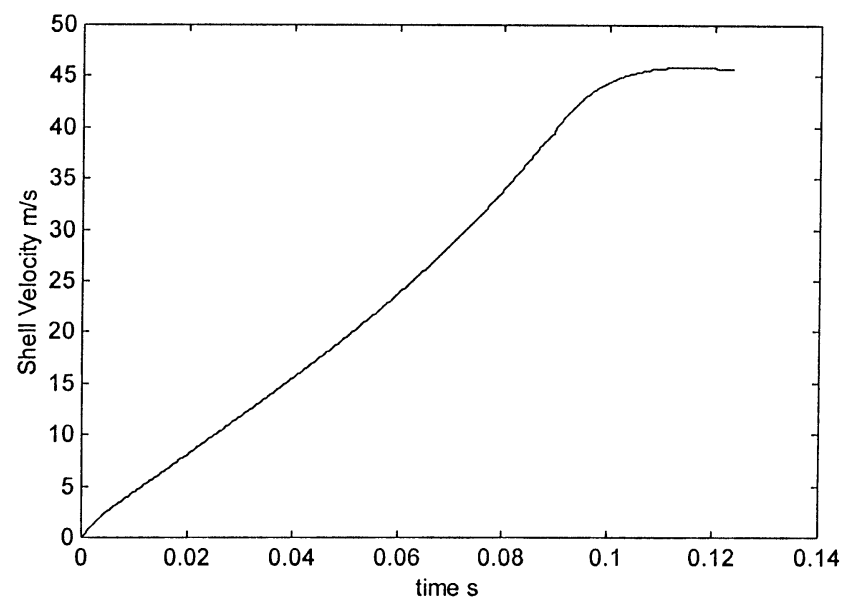

Fig. 6. Flight velocity of a waterjet rocket for the first $0.12 \mathrm{sec}$.

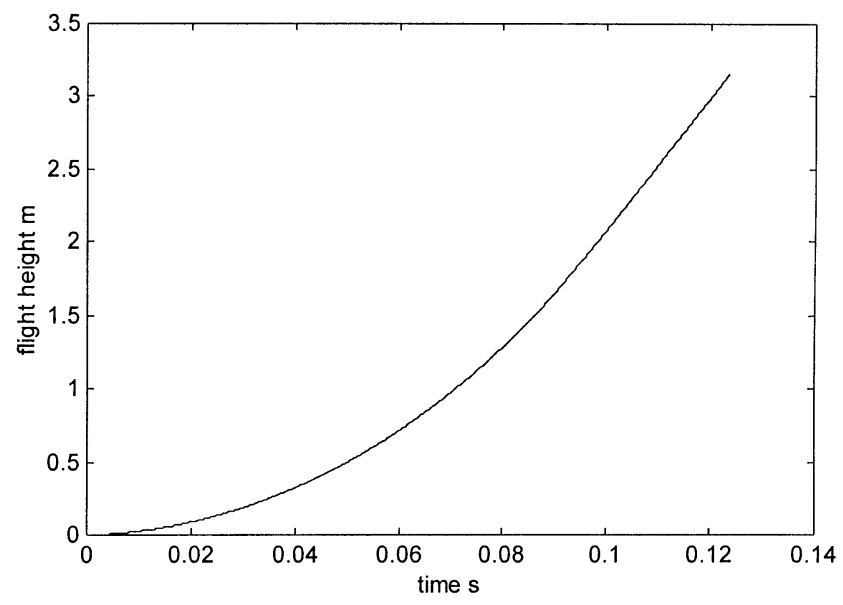

Fig. 7. Flight height histogram of a waterjet rocket for the first $0.12 \mathrm{sec}$.

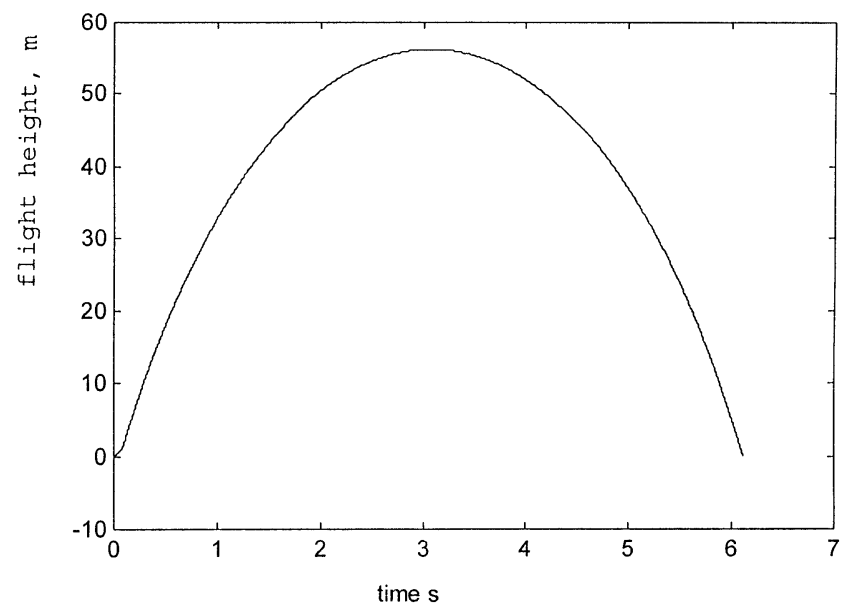

Fig. 8. Whole flight histogram of a waterjet rocket.

PC with Intel Pentium $233 \mathrm{cpu}$. Under the above given conditions, the waterjet rocket will finish its propulsion stage in about $0.1 \mathrm{sec}$; the flight velocity and height histograms for that duration are shown in Figs. 6 and 7, respectively, and the whole flight height histogram is shown in Fig. 8.

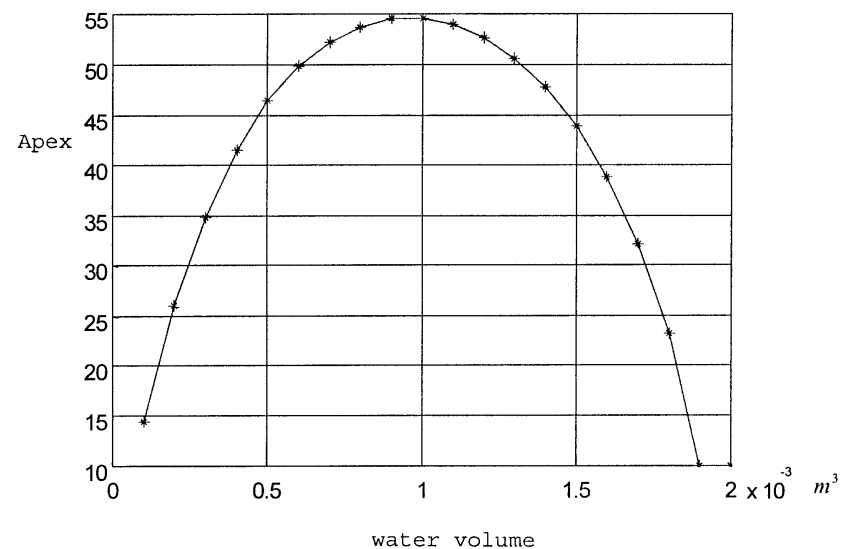

Fig. 9. Apex vs. water volume in the waterjet rocket.

It is noteworthy that for higher internal air pressure, the curves in Fig. 5 shift somewhat to the right; thus for higher air pressure, the optimal apex will correspond to a heavier shell mass. Alternatively, an overly heavy shell mass will not attain a higher apex generally because of a smaller maximum velocity being attained during the propulsion stage. After all the water inside a waterjet rocket has been just expelled, there is still much air pressure left in the rocket. Although the remaining air usually weighs only a few grams, its impulse effect to enhance the $V_{\text {cv }}$ postwaterjet stage should be somehow taken into consideration. For a pure pressurized air rocket with about $250 \mathrm{kpa}$ (gauge) and shell mass of about $0.255 \mathrm{~kg}$, associated experiments show that the impulse due to pure air expulsion will be approximately equivalent to a $6 \mathrm{~m} / \mathrm{sec}$ increase to the maximum $V_{\mathrm{cv}}$. In this study, the effect of pure air expulsion is generally considered in the apex prediction of the waterjet rocket.

Furthermore, when we change the air/water ratio, the flight height will change accordingly, because less air means less energy source and less water means less usable "pure" propellant. Nevertheless, more water means a heavier rocket with severe gravity effect. Thus the flight apex of a waterjet rocket is a science of compromise. Figure 9 indicates that an optimal height corresponds to a water volume of about $0.9 l$ under other conditions given above. This situation is generally agreeable also with the experimental results.

\section{Conclusions}

For accommodating the integrated energy method for a propulsion dynamics analysis of the waterjet rocket, the generalized power equation has been derived herein by a more efficient interweaved scheme than ever, ${ }^{8)}$ with the aid of the lagrangian Reynolds transport equation. ${ }^{8,9)}$ This generalized power equation involves a complete spectrum of physical parameters and is invariant to any unaccelerating observer. Since its conventional counterpart with only one or two terms will give sizable offset in computation, ${ }^{10)}$ this novel generalized power Eq. (7) is a crucial ingredient in the above integrated energy method for a waterjet rocket. 
In this paper, the propulsion dynamics of a waterjet rocket is analyzed by solving the momentum and new generalized total kinetic power equations simultaneously by computational procedure with the aid of Matlab. We can then manage to obtain the instant $\dot{V}_{\text {cv }}$ and $\dot{v}_{\mathrm{e}}$, and convolutionally we can solve the whole flight histogram of the waterjet rocket. This integrated energy approach synthesizes both the internal and external dynamics analyses of the waterjet rocket. Moreover, it elegantly makes use of the predictable power supply in waterjet rocket. And for better apex prediction, the effect of pure air expulsion postwaterjet is generally considered for the rocket. In the last section, the effects due to shell mass variation, internal air pressure, and air/water ratio are explicitly expressed in figures, and a sampled flight histogram of the given rocket is also displayed. Results of the theoretical analysis by the above integrated scheme are generally agreeable with the experimental flight apex and thus demonstrate the validity of this integrated energy method. The slight discretion of a few percent is understandable, considering especially the complicated internal/external flow field of the rocket, some minor launch energy loss, and environmental effects.

The waterjet rocket makes use of high pressure air as its power source. Compared with traditional rockets, it is characterized with (1) nature (uses water and air), (2) much simpler structure, (3) no combustion (safe and green), (4) separately stored and adjustable energy and propellant mass in contrast to conventional propellant, (5) interesting (6) minimal cost involved in acquisition and launch. Therefore the waterjet rocket is a very good science model for propulsion analysis, experiment, and education because of the abundance of very easily adjustable key parameters, such as internal air pressure, shell mass and diameters, air/water proportion, external shape and associated $C_{\mathrm{d}}$, launch angle, and even size and shape of the nozzle. Incidentally, the waterjet may have the potential as the power source for a rescue rocket, or a short-range missile, such as an antitank missile, with no smoke or heat to be detected (since there is no firing of a waterjet rocket). In conclusion, while simultaneous equations of the momentum and the generalized rocket total kinetic power involve more physical parameters to be manipulated, that means there will be wider room in quest of better rocket propulsion performance, ${ }^{11-13)}$ especially through the heuristic analysis, design, and experiment of the versatile but affordable waterjet rocket.

\section{References}

1) Mahulikar, S. P.: Philosophical Approach to the Basic Understanding of the Mechanics of Jet Propulsion, SAE Paper No. 920960, 1992, pp. 1-7.

2) Sutton, G. P.: Rocket Propulsion Elements, 6th ed., John Wiley, New York, 1967.

3) Hill, P. and Peterson, C.: Mechanics and Thermodynamics of Propulsion, 2nd ed., Addison Wesley, New York, 1992.

4) Lodge, S.: Model Rocketry, Traplet Publications Ltd., Worcestershire, $\mathrm{UK}, 1996$.

5) Mazubi, K., Sukiyama, W. K. and Yamakawa, C. K.: Making of PET-Bottle Rocket, 1st ed., Eichi Publication Co., Tokyo, 1996 (in Japanese).

6) Tsiolkovskiy, K. E.: Works on Rocket Technology, NASA Technical Translation, TT F-243, NASA, Washington, D.C., 1965.

7) Barrere, M. A., Jaumotte, A., Deveubeke, B. F. and Vandenkerckhove, J.: Rocket Propulsion, 1st ed., Van Nostrand, New York, 1959.

8) Lee, H. J. and Lee, H. W.: Deriving the Generalized Rocket Kinetic Power Equations and Associated Propulsion Indexes, JSME Int. J., 42 (1999), pp. 127-136.

9) Lee, H. J. and Huang, S. C.: On the Derivation Process of Reynolds Transport Equation, Int. J. Mech. Eng. Educ., 21 (1993), pp. 49-53.

10) Lee, H. J. and Chang C. L.: Deriving the Generalized Power and Efficiency Equations for Jet Propulsion Systems, JSME Int. J., 44 (2001), in press.

11) Tsien H. S. and Evens, R. C.: Optimal Thrust Programming for a Sounding Rocket, J. Am. Rocket Soc., 21 (1951), pp. 635-643.

12) King, M. K.: Rocket Propulsion Strategy Based on Kinetic Energy Management, J. Propul. Power, 14 (1998), pp. 270-272.

13) Sparenberg, J. A.: Hydrodynamic Propulsion and Its Optimization, Kluwer Academic Publishers, Boston, 1995.

\section{Appendix}

For Matlab program for propulsion dynamics analysis of waterjet rocket, Please see the end of Web site: http://www.yamtopia.com.tw/home/microprop/ 